International Mathematical Forum, Vol. 9, 2014, no. 34, 1659 - 1667

HIKARI Ltd, www.m-hikari.com

http://dx.doi.org/10.12988/imf.2014.49155

\title{
Characterization of Weakly Primary Ideals over Non-commutative Rings
}

\author{
Arwa Eid Ashour \\ Department of Mathematics, The Islamic University of Gaza \\ Gaza, Palestine, P.O. Box 108 \\ Mohammad Hamoda \\ Department of Mathematics, Al-Aqsa University \\ Gaza, Palestine, P.O. Box 4015
}

Copyright (c) 2014 Arwa Eid Ashour and Mohammad Hamoda. This is an open access article distributed under the Creative Commons Attribution License, which permits unrestricted use, distribution, and reproduction in any medium, provided the original work is properly cited.

\begin{abstract}
In this paper, we introduce the concept of weakly primary ideals over non-commutative rings. Several results on weakly primary ideals over non-commutative rings are proved. We prove that a right (resp. left) weakly primary ideal $P$ of a ring $\mathrm{R}$ that is not right (resp. left) primary satisfies $P^{2}=0$. We give useful characterization of weakly primary ideals over non-commutative rings with nonzero identities. We prove that every irreducible ideal of a right (resp. left) Noetherian ring $R$ is right (resp. left) weakly primary ideal in $R$.
\end{abstract}

Mathematics Subject Classification: Primary 16D25, Secondary 16D80, $16 \mathrm{~N} 60$

Key words:Prime ideal, primary ideal, weakly primary ideal, Jacobson radical, right weakly primary ideal, left weakly primary ideal

\section{Introduction}

Recently, extensive researches have been done on prime and primary ideals and submodules. D.D. Anderson and E. Smith [1] defined a proper ideal $P$ of 
a commutative $\operatorname{ring} R$ with identity to be weakly prime if $0 \neq a b \in P$ implies $a \in P$ or $b \in P$. The concept of weakly primary ideals over commutative rings has been studied by E.E. Atani and F. Farzalipour [2]. They defined a weakly primary ideal as a proper ideal $P$ over a commutative ring $R$ with the property that if $0 \neq a b \in P$, then $a \in P$ or $b^{n} \in P$ for some positive integer $n$. The structure of weakly prime ideals over non-commutative rings has been studied by Y. Hirano, E. Poon, and H. Tsutsui in [6]. Also, they investigated the structure of rings, not necessarily commutative nor with identity, in which all ideals are weakly prime. Various properties of weakly primary (weakly prime) subtractive ideals over commutative semirigs have been studied in [3] and [5]. The motivation of this paper is to continue the studying of the family of primary ideals, also to extend the results of Anderson [1], Atani and Frazalipour [2], and Hirano, Poon, and Tsutsui [6] to the weakly primary ideals over noncommutative rings.

Throughout this paper, all rings are assumed to be associative with nonzero identities, and all modules are unital. By "an ideal" we mean a 2-sided ideal.

\section{Preliminary Notes}

In this section we give some basic definitions:

Definition 2.1 [7] A proper ideal $P$ of a ring $R$ is called prime if for ideals $A, B$ of $R$ with $A B \subseteq P$, then $A \subseteq P$ or $B \subseteq P$.

Definition 2.2 [6] A proper ideal $P$ of a ring $R$ is called weakly prime if for ideals $A, B$ of $R$ with $0 \neq A B \subseteq P$, then $A \subseteq P$ or $B \subseteq P$.

Definition 2.3 [9] The prime radical of a ring $R$ denoted by $P(R)$ is the intersection of all prime ideals of $R$. If $R$ has no prime ideals, then $P(R)=R$.

Definition 2.4 [9] The nil radical of a ring $R$ denoted by $N(R)$ is the sum of all nil ideals of $R$.

Definition 2.5 [7] The Jacobson radical of a ring $R$ denoted by $J(R)$ is the intersection of all maximal right (or left) ideals of $R$.

Note that from the above definition, we can see that $J(R)$ is the set of all elements $x \in R$ such that $y x+1$ is a unit of $R$ for all $y \in R$. (see [4]).

Definition 2.6 [8] Let $R$ be a ring. A nonempty set $S \subseteq R$ is called an $m$-system if for any $a, b \in S$, there exists $r \in R$ such that arb $\in S$. 


\section{Results and Discussion}

We begin by the following two definitions:

Definition 3.1 Let $R$ be a ring. A proper ideal $P$ of $R$ is called right primary if whenever $A, B$ are ideals of $R$ such that $A B \subseteq P$, then $A \subseteq P$ or $B^{n}=\left\{b^{n}: b \in B\right\} \subseteq P$ for some $n \in N$. A proper ideal $P$ of $R$ is called left primary if whenever $A, B$ are ideals of $R$ such that $A B \subseteq P$, then $B \subseteq P$ or $A^{n}=\left\{a^{n}: a \in A\right\} \subseteq P$ for some $n \in N$.

The ideal $P$ is called primary if it is both right and left primary.

Definition 3.2 Let $R$ be a ring. A proper ideal $P$ of $R$ is called right weakly primary if whenever $A, B$ are ideals of $R$ such that $0 \neq A B \subseteq P$, then $A \subseteq P$ or $B^{n} \subseteq P$ for some $n \in N$. A proper ideal $P$ of $R$ is called left weakly primary if whenever $A, B$ are ideals of $R$ such that $0 \neq A B \subseteq P$, then $B \subseteq P$ or $A^{n} \subseteq P$ for some $n \in N$.

The ideal $P$ is called weakly primary if it is both right and left weakly primary.

\section{Remarks 3.1}

(1) The sets $A^{n}$ and $B^{n}$ in the above definitions are not necessarily ideals in a ring $R$.

(2) Clearly every primary ideal of a ring $R$ is weakly primary. However, since 0 is always weakly primary (by definition), a weakly primary ideal need not be primary. Thus the weakly primary ideal concept is a generalization of the concept of primary ideal.

(3) It's easy to see that every weakly prime ideal is a weakly primary, however the converse is not in general true.

\section{Example 3.1}

(1) Let $R=Z \times Z$. The ideal $P=2 Z \times 0$ is a weakly prime ideal of $R$. However, $4 Z \times 0$ is a weakly primary ideal of $R$, that is not weakly prime ideal of $R$.

(2) Let $R=Z_{12}$ and let $P=\{0,6\}$. Then $P$ neither primary nor weakly primary ideal.

Definition 3.3 [8] Let $P$ be a proper ideal of a ring $R$. Then the set

$$
\{r \in R \text { : every } m-\text { system containing } r \text { meets } P\}
$$

is an ideal of $R$ called the radical of the ideal $P$ on $R$ denoted by $\operatorname{Rad}_{R}(P)$.

Note: It is easy to see that $\operatorname{Rad}_{R}(P)$ equals the intersection of all prime ideals of $R$ containing $P$.

Remarks 3.2

(i) Denote the set $\left\{r \in R: r^{n} \in P\right.$ for some $\left.n \in N\right\}$ by $\sqrt{P}$. Then

$$
\operatorname{Rad}_{R}(P)=\{r \in R: \text { every } m \text { - system containing } r \text { meets } P\}
$$




$$
\subseteq \sqrt{P}=\left\{r \in R: r^{n} \in P \text { for some } n \in N\right\} \text {, see [8]. }
$$

(ii) If $R$ is a commutative ring, one can check that the inclusion " $\subseteq$ " above is actually an equality.

Theorem 3.4 Let $R$ be a ring and let $P$ be a proper ideal of $R$.

(i) If for all $a, b \in R, 0 \neq a b \in P \Rightarrow a \in P$ or $b^{n} \in P$ for some $n \in I$, where $I$ is a finite subset of $N$. Then $P$ is a right weakly primary ideal of $R$. (ii) If for all $a, b \in R, 0 \neq a b \in P \Rightarrow b \in P$ or $a^{n} \in P$ for some $n \in I$, where $I$ is a finite subset of $N$. Then $P$ is a left weakly primary ideal of $R$.

Proof:(i) Let $P$ be a proper ideal of $R$ and let $A$ and $B$ are ideals of $R$ such that $0 \neq A B \subseteq P$ and $A \nsubseteq P$, then there exists an element $a \in A-P$. And for every element $b \in B, 0 \neq a b \in A B \subseteq P$. Since $a \notin P$, then $\forall b \in B, b^{n} \in P$, for some $n \in I$, where $I$ is a finite subset of $N$. Now since $I$ is a finite subset of $N$, let $m=\max \left\{n: b^{n} \in P, n \in I, b \in B\right\}$. So $B^{m} \subseteq P$. Therefore $P$ is a right weakly primary ideal of $R$.

(ii) Proceed similar as in (i).

Remark 3.3 The converse of Theorem 3.4 is true when $R$ is commutative. To see this, let $P$ be a right weakly primary ideal of $R$ and let $a, b \in R$ with $0 \neq a b \in P$, then the principal ideal $\langle a b\rangle$ is contained in $P$ (by definition). So commutativity of $R$ implies that $0 \neq<a><b>\subseteq<a b>\subseteq P \Rightarrow<$ $a>\subseteq P$ or $\left\langle b>^{n} \subseteq P\right.$ for some $n \in N$. Whence $a \in P$ or $b^{n} \in P$ for some $n \in N$. Similarly, if $P$ is a left weakly primary ideal of $R$, then $a, b \in R, 0 \neq a b \in P \Rightarrow b \in P$ or $a^{n} \in P$ for some $n \in N$.

Here is a very useful characterization of weakly primary ideals:

Proposition 3.5 Let $R$ be a ring and let $P$ be a proper ideal of $R$.

(i) $P$ is a right weakly primary ideal iff when $a, b \in R$ with $0 \neq a R b \subseteq P$, then $a \in P$ or $b^{n} \in P$ for some $n \in I$, where $I$ is a finite subset of $N$.

(ii) $P$ is a left weakly primary ideal iff when $a, b \in R$ with $0 \neq a R b \subseteq P$, then $b \in P$ or $a^{n} \in P$ for some $n \in I$, where $I$ is a finite subset of $N$.

Proof:(i) Let $0 \neq a R b \subseteq P$, and suppose that $a \notin P$. This yields $R a R b R \subseteq R P R \subseteq P$ since $P$ is an ideal of $R$. Now $0 \neq R a R . R b R \subseteq R a R b R \subseteq P$. Let $A=R a R$ and $B=R b R$. Then $0 \neq A B$ with $A \nsubseteq P$. Since $A$ and $B$ are ideals of $R$ and $P$ is right weakly primary, then for all $b \in B, b^{n} \in P$ for some $n \in I$, where $I$ is a finite subset of $N$. The required converse is immediate from Theorem 3.4 .

(ii) Proceed similar as in (i).

Proposition 3.6 Let $R$ be a ring and let $P$ be a proper ideal of $R$.

(i) If $P$ is a right weakly primary ideal. Then $P$ satisfies the following condition:

$\forall a, b \in R$ with $0 \neq a R b \subseteq P$, then $a \in P$ or $b^{n} \in P$ for some $n \in N$. 
(ii) If $P$ is a left weakly primary ideal. Then $P$ satisfies the following condition: $\forall a, b \in R$ with $0 \neq a R b \subseteq P$, then $b \in P$ or $a^{n} \in P$ for some $n \in N$.

Proof: Forward by using the proof of Proposition 3.5.

Now, the following proposition is easy to prove:

Proposition 3.7 Let $R$ be a ring, let $P$ be an ideal of $R$, and let $x \in R-\sqrt{P}$.

(i) The set $(P: x R)=\{y \in R: x R y \subseteq P\}$ is a right ideal of $R$ containing $P$.

(ii) The set $(P: R x)=\{y \in R: y R x \subseteq P\}$ is a left ideal of $R$ containing $P$.

Remark 3.4 If $R$ is a commutative ring with identity and $P$ is an ideal of $R$, then it is easy to see that $\forall x \in R-P$, the set $(P: x)=\{y \in R: y x \in P\}$ is an ideal of $R$.

Theorem 3.8 Let $R$ be a ring, let $P$ be a proper ideal of $R$ and let $x \in R-\sqrt{P}$. If $P$ is a right weakly primary ideal of $R$, then $(P: x R) \subseteq \sqrt{P} \cup(0: x R)$. Proof: Assume that $P$ is a right weakly primary ideal of $R$ and let $y \in(P$ : $x R$ ) with $x \notin \sqrt{P}$. Then $x R y \subseteq P$ and $x^{n} \notin P \forall n \in N \Rightarrow x \notin P$. If $x R y=0$, then $y \in(0: x R)$. If $x R y \neq 0$, then $P$ right weakly primary gives $y^{n} \in P$ for some $n \in N$ (see Proposition 3.6) Hence $y \in \sqrt{P}$. Therefore $(P: x R) \subseteq \sqrt{P} \cup(0: x R)$.

Similarly the left analogues of Theorem 3.8 can be established.

Lemma 3.9(i) Let $P$ be a right weakly primary ideal of a ring $R$. If $P$ is not a right primary ideal, then $P^{2}=0$.

(ii) Let $P$ be a left weakly primary ideal of a ring $R$. If $P$ is not a left primary ideal, then $P^{2}=0$.

Proof:(i) Suppose that $P^{2} \neq 0$; we want to show that $P$ is a right primary ideal of $R$. Let $a, b \in R$ such that $a R b \subseteq P$. If $a R b \neq 0$, then $a \in P$ or $b^{n} \in P$ for some $n \in N$ (by Proposition 3.5.) So assume that $a R b=0$. If $0 \neq a P \subseteq P$, then there is an element $d$ of $P$ such that $a d \neq 0$. Hence $0 \neq a R d=a R(d+b) \subseteq P$. Then either $a \in P$ or $(b+d)^{n} \in P$ for some $n \in N \Rightarrow a \in P$ or $b^{n} \in P$ for some $n \in N$. Therefore $P$ is a right primary ideal. Now we can assume that $a P=0$. If $P b \neq 0$, then there exists $u \in P$ such that $u b \neq 0$. Now $0 \neq u R b=(u+a) R b \subseteq P$. So $a \in P$ or $b^{n} \in P$ for some $n \in N$ and hence $P$ is a right primary ideal. Thus we can assume that $P b=0$. Since $P^{2} \neq 0$, there are elements $e, f \in P$ such that $e f \neq 0$. Then $0 \neq e R f=(a+e) R(b+f) \subseteq P$, so either $a \in P$ or $b^{n} \in P$ for some $n \in N$ and hence $P$ is a right primary ideal.

(ii) Proceed similar as in (i).

Corollary 3.10(i) If $P$ is a right weakly primary ideal of a ring $R$ that is not right primary then $\sqrt{P}=\sqrt{0}$.

(ii) If $P$ is a left weakly primary ideal of a ring $R$ that is not left primary then $\sqrt{P}=\sqrt{0}$.

Proof:(i) Assume that $P$ is a right weakly primary ideal that is not right primary over the ring $R$, then it is clear that $\sqrt{0} \subseteq \sqrt{P}$. Now by Lemma 3.9, $P^{2}=0$ gives $P \subseteq \sqrt{0}$, hence $\sqrt{P} \subseteq \sqrt{0}$. Therefore $\sqrt{P}=\sqrt{0}$. 
(ii) Proceed similar as in (i).

In general the intersection of a family of right (resp. left) primary ideals is not right (resp. left) primary, but we have the following result:

Theorem 3.11(i) Let $\left\{P_{i}\right\}_{i \in I}$ be a family of right weakly primary ideals of a ring $R$ that are not right primary. Then $\bigcap_{i \in I} P_{i}$ is a right weakly primary ideal of $R$.

(ii) Let $\left\{P_{i}\right\}_{i \in I}$ be a family of left weakly primary ideals of a ring $R$ that are not left primary. Then $\bigcap_{i \in I} P_{i}$ is a left weakly primary ideal of $R$.

Proof:(i) Assume that $P_{i}$ is a right weakly primary ideal of $R$ that is not right primary $\forall i \in I$. First, want to show that $\sqrt{\bigcap_{i \in I} P_{i}}=\bigcap_{i \in I} \sqrt{P_{i}}$.

$\sqrt{\bigcap_{i \in I} P_{i}}=\left\{r \in R: r^{n} \in \bigcap_{i \in I} P_{i}\right.$ for some $\left.n \in N\right\}$

$=\left\{r \in R: r^{n} \in P_{i} \forall i \in I\right.$ and some $\left.n \in N\right\}$. Thus

$\sqrt{\bigcap_{i \in I} P_{i}} \subseteq \bigcap_{i \in I} \sqrt{P_{i}}$. For the other inclusion, suppose that $r \in \bigcap_{i \in I} \sqrt{P_{i}}$. Since $\forall i, \sqrt{P_{i}}=\sqrt{0}$ by corollary 3.10(i), then $\bigcap_{i \in I} \sqrt{P_{i}}=\sqrt{0}$. Thus $r \in \sqrt{0}$ implies $r^{m}=0$ for some $m \in N$. So $r^{m} \in P_{i} \forall i \in I$, and hence $r \in \sqrt{\bigcap_{i \in I} P_{i}}$. Now $\bigcap_{i \in I} P_{i}$ is a proper ideal of $R$ because $\sqrt{\bigcap_{i \in I} P_{i}}=\sqrt{0} \neq R$. Suppose that $a, b \in R$ such that $0 \neq a R b \subseteq \bigcap_{i \in I} P_{i}$ but $a \notin \bigcap_{i \in I} P_{i}$. Then there exists an element $j \in I$ such that $a \notin P_{j}$ and $a R b \subseteq P_{j}$. It follows that $b^{n} \in P_{j}$ for some $n \in N$ i.e. $b \in \sqrt{P_{j}}=\sqrt{0}=\sqrt{\bigcap_{i \in I} P_{i}}$. So $b^{n} \in \bigcap_{i \in I} P_{i}$ for some $n \in N$ and hence $\bigcap_{i \in I} P_{i}$ is a right weakly primary ideal of $R$.

(ii) Proceed similar as in (i).

Next, we state and prove a version of Nakayama's Lemma.

Theorem 3.12 Let $P$ be a right weakly primary ideal of a ring $R$ that is not right primary. Then the following hold:

(i) $P \subseteq J(R)$.

(ii) If $A$ is a right $R$-module and $A P=A$, then $A=0$.

(iii) If $A$ is a right $R$-module and $N$ is a submodule of $A$ such that $A P+N=A$, then $A=N$.

Proof:(i) Let $a \in P$. We may assume that $a \neq 0$. It is sufficient to show that $b a+1$ is a unit of $R$ for every $b \in R$. Since $P$ is a right weakly primary ideal that is not right primary over $R$, so $P^{2}=0$, (by Lemma 3.9) $\Rightarrow$ $1=(1+b a)(1-b a) \Rightarrow 1+b a$ is a unit of $R \Rightarrow a \in J(R)$.

(ii) Since $A P=A$ and $P^{2}=0$ (by Lemma 3.9), we have $0=A P^{2}=A P$.

(iii) Given $A P+N=A$ implies that $A P^{2}+N P=A P$, i.e. $N P=A P$ which implies that $N P+N=A P+N$, hence $N=A P+N=A$.

Similarly the left analogues of Theorem 3.12 can be established.

In the following proposition, we investigate the structure of rings, not necessarily commutative in which all proper ideals are right (or left) weakly primary.

Proposition 3.13(i) Every proper ideal of a $\operatorname{ring} R$ is a right weakly primary ideal iff for any ideals $I$ and $J$ of $R, I J=I$ or $J^{n} \subseteq I J$ for some $n \in N$ or 
$I J=0$.

(ii) Every proper ideal of a ring $R$ is a left weakly primary ideal iff for any ideals $I$ and $J$ of $R, I J=J$ or $I^{n} \subseteq I J$ for some $n \in N$ or $I J=0$.

Proof:(i) Suppose that every proper ideal of a $\operatorname{ring} R$ is a right weakly primary. Let $I$ and $J$ be ideals of $R$. If $I J \neq R$, then $I J$ is a right weakly primary. If $0 \neq I J \subseteq I J$, then we have $I \subseteq I J$ or $J^{n} \subseteq I J$ for some $n \in N$ that is $I=I J$ or $J^{n} \subseteq I J$ for some $n \in N$. If $I J=R$, then we have $I=J=R$. Conversely, let $K$ be any proper ideal of $R$ and assume that $0 \neq I J \subseteq K$ for ideals $I$ and $J$ of $R$. Then we have either $I=I J \subseteq K$ or $J^{n} \subseteq I J \subseteq K$ for some $n \in N$.

(ii) Proceed similar as in (i).

Notation: We denote $R_{n \times n}=\left\{\left[a_{i j}\right]: a_{i j} \in R\right\}$, the set of $n \times n$ matrices over a ring $R$. Under addition and multiplication of matrices, $R_{n \times n}$ forms a non-commutative ring with identity. $A$ is an ideal in $R_{n \times n}$ if and only if $A=P_{n \times n}$, the set of $n \times n$ matrices over an ideal $P$ in $R$.

Proposition 3.14 Let $R$ be a ring, let $P$ be a proper ideal of $R$.

(i) If $P_{n \times n}$ is a right weakly primary ideal of $R_{n \times n}$, then $P$ is a right weakly primary ideal of $R$.

(ii) If $P_{n \times n}$ is a left weakly primary ideal of $R_{n \times n}$, then $P$ is a left weakly primary ideal of $R$.

Proof:(i) Let $a, b \in R$ such that $0 \neq a R b \subseteq P$. As $a E_{11} \in R_{n \times n}$ and $b E_{11} \in R_{n \times n}$, where $E_{i j}$ is the matrix in $R_{n \times n}$ with $e_{i j}=1$ and 0 otherwise. Then $0 \neq a E_{11} R_{n \times n} b E_{11} \subseteq P_{n \times n}$. Hence $a E_{11} \in P_{n \times n}$ or $\left(b E_{11}\right)^{m} \in P_{n \times n}$ for some $m \in I, I$ is a finite subset of $N$, by Proposition 3.5. Now $a \in P$ or $b^{m} \in P$ for some $m \in I, I$ is a finite subset of $N$. Therefore $P$ is a right weakly primary ideal of $R$.

(ii) Proceed similar as in (i).

Example 3.2 Let $R=(Z,+,$.$) and consider the ring$

$H=\left\{\left(\begin{array}{ll}a & b \\ 0 & c\end{array}\right): a, b, c \in R\right\}$.

Let $P$ be an ideal of $R$. Then $T=\left\{\left(\begin{array}{ll}a & b \\ 0 & c\end{array}\right): a, b, c \in P\right\}$ is an ideal of $H$. Let $A=\left(\begin{array}{ll}2 & 0 \\ 0 & 1\end{array}\right)$ and $B=\left(\begin{array}{ll}1 & 0 \\ 0 & 2\end{array}\right)$ are in $H$

$P=<2>\Rightarrow \neq A H B \subseteq T$, but $A, B \notin T$ and $A^{n} \notin T, \forall n \in N, B^{m} \notin T$, $\forall m \in N$. Hence $T$ is not a weakly primary ideal of $H$.

Theorem 3.15(i) Let $R$ be a right Noetherian ring. Then every irreducible ideal of $R$ is right weakly primary ideal in $R$.

(ii) Let $R$ be a left Noetherian ring. Then every irreducible ideal of $R$ is left weakly primary ideal in $R$.

Proof:(i) Let $P$ be an irreducible ideal of $R$, and let $a, b \in R$ with $0 \neq a R b \subseteq P$. Clearly $(P: b) \subseteq\left(P: b^{2}\right) \subseteq\left(P: b^{3}\right) \subseteq \cdots \cdots$ is an ascending 
chain of ideals in $R$, where $(P: b)=\{r \in R: b r \in P\}$ is an ideal in $R$. Thus there exists $n \geq 1$ such that $\left(P: b^{n}\right)=\left(P: b^{k}\right)$ for all $k \geq n$. Let $A=P+a R$ and $B=P+b^{n} R, n \in I, I$ is a finite subset of $N$. We claim that $A \cap B=P$. Clearly $P \subseteq A \cap B$. Let $x \in A \cap B$. Then $x=a_{1}+a r_{1}$ and $x=a_{2}+b^{n} r_{2}$ for some $a_{1}, a_{2} \in P$ and $r_{1}, r_{2} \in R$. It can shown easily that if $y \in P$ then $b^{t} y \in P \forall t \geq 1$. Now $b^{n+1} x=b^{n+1} a_{1}+b^{n+1} a r_{1}=b^{n+1} a_{1}+b^{n} b a r_{1} \in P$. Thus $b^{n+1} a_{2}+b^{n+1+n} r_{2}=b^{n+1}\left(a_{2}+b^{n} r_{2}\right)=b^{n+1} x \in P$. Also $b^{n+1} a_{2} \in P$. Hence $b^{n+1+n} r_{2} \in P$. Therefore $r_{2} \in\left(P: b^{n+n+1}\right)=\left(P: b^{n}\right)$. Therefore $b^{n} r_{2} \in P$. Thus $x=a_{2}+b^{n} r_{2} \in P$. Now $A \cap B=P$. Thus $A=B$ or $B=P$ since $P$ is irreducible ideal. Hence $a \in P$ or $b^{n} \in P$ for some $n \in I, I$ is a finite subset of $N$. Hence $P$ is right weakly primary ideal in $R$.

(ii) Proceed similar as in (i).

Proposition 3.16 Let $I \subseteq P$ be proper ideals of a ring $R$.

(i) If $P$ is a right weakly primary ideal, then $P / I$ is a right weakly primary ideal.

(ii) If $P$ is a left weakly primary ideal, then $P / I$ is a left weakly primary ideal. Proof:(i) Assume that $P$ is a right weakly primary ideal of $R$ and let $0 \neq$ $(a+I) R(b+I) \subseteq P / I$, where $a, b \in R$. Since $(a+I) R(b+I)=a R b+I \subseteq P / I$, then $a R b \subseteq P$. If $a R b=0$, then $(a+I) R(b+I)=I$, a contradiction. So if $P$ is a right weakly primary ideal of $R$, then by Proposition 3.5, $a \in P$ or $b^{n} \in P$ for some $n \in I$, where $I$ is a finite subset of $N$. Hence $(a+I) \in P / I$ or $\left(b^{n}+I\right)=(b+I)^{n} \in P / I$ for some $n \in I$, where $I$ is a finite subset of $N$. Therefore $P / I$ is a right weakly primary ideal.

(ii) Proceed similar as in (i).

Acknowledgements. The authors thanks the referee for useful comments.

\section{References}

[1] D.D.Anderson and E.Smith, Weakly Prime Ideals, Houston J. Math, 29 4, (2003), 831-840.

[2] S.E.Atani and F.Frazalipour, On Weakly Primary Ideals, Georgian Mathematical Journal, 12 3, (2005), 423-429.

[3] S.E.Atani, On $k$-Weakly Primary Ideals over Semirings, Sarajevo Journal of Mathematics, 3 15, (2007), 9-13.

[4] E.H.Feller, Properties of Primary Noncommutative Rings, University of Wisconsin-Milwaukee, 89, (1958), 79-91. http://dx.doi.org/10.1090/s0002-9947-1958-0098763-0 
[5] V.Gupta and J.N.Chaudhari, Characterization of Weakly Prime Subtractive Ideals in Semirings, Bulletin of Institute of Mathematics-Academia Sinica, 3 3, (2008), 347-352.

[6] Y.Hirano, E.Poon and H.Tsutsui, On Rings in Which Every Ideal is Weakly Prime, Bull.Korean Math.Soc, 47 5, (2010), 1077-1087. http://dx.doi.org/10.4134/bkms.2010.47.5.1077

[7] Thomas W.Hungerford, Algebra, Springer, New York, (1973).

[8] T.Y.Lam, A first Course in Noncommutative Rings, Graduate Texts in Mathematics, Springer-Verlag, Berlin-Heidelberg-New Yourk, (1991). http://dx.doi.org/10.1007/978-1-4684-0406-7

[9] R.Wisbauer, Foundations of Module and Ring Theory. University of Duesseldorf, Gordon and Breach Science Publishers, (1991).

Received: September 8, 2014; Published: November 12, 2014 